\title{
Many Polytopes Meeting the Conjectured Hirsch Bound
}

\author{
F. B. Holt ${ }^{1}$ and V. Klee ${ }^{2}$ \\ ${ }^{1}$ The Boeing Company, P.O. Box 3707, \\ M/S 7L-21 Seattle, WA 98124-2207, USA \\ fred.b.holt@boeing.com \\ ${ }^{2}$ Department of Mathematics, University of Washington, \\ Box 354350, Seattle, WA 98195-4350, USA \\ klee@math.washington.edu
}

Dedicated to Jörg Wills on the occasion of his 60th birthday

\begin{abstract}
The still open Hirsch conjecture asserts that $\Delta(d, n) \leq n-d$ for all $n>d \geq 2$, where $\Delta(d, n)$ denotes the maximum edge-diameter of (convex) $d$-polytopes with $n$ facets. This paper adds to the list of pairs $(d, n)$ that are known to be $H$-sharp in the sense that $\Delta(d, n) \geq n-d$. In particular, it is proved that $\Delta(d, n) \geq n-d$ for all $n>d \geq 14$.
\end{abstract}

\section{Introduction}

For two vertices $x$ and $y$ of a polytope $P$, the distance $\delta_{P}(x, y)$ is defined as the smallest number of edges of $P$ that can be used to form a path from $x$ to $y$. The diameter $\delta(P)$ of $P$ is the maximum of $\delta_{P}(x, y)$ over all pairs $(x, y)$ of $P$ 's vertices. As reported by Dantzig [D1], [D2], W. M. Hirsch conjectured in 1957 that $\Delta(d, n) \leq n-d$ for all $n>d \geq 2$, where $\Delta(d, n)$ is the maximum diameter of $d$-polytopes with $n$ facets. The purpose of the present paper is to enlarge the set $\mathcal{S}$ of pairs $(d, n)$ that are known to be $H$-sharp for the Hirsch conjecture, in the sense that $\Delta(d, n) \geq n-d$. It has long been known that $\mathcal{S}$ includes all pairs $(d, n)$ with $d<n \leq 2 d$, and that when $d \leq 3$ the condition $n \leq 2 d$ is also necessary for $H$-sharpness [K]. Hence we focus on pairs $(d, n)$ for which $d \geq 4$ and $n>2 d$.

We use the term $(d, n)$-polytope to denote a simple $d$-polytope with precisely $n$ facets. It is known that $\Delta(d, n)$ is attained as $\delta_{P}(x, y)$ for some $(d, n)$-polytope $P$ and two vertices $x$ and $y$ of $P$, and that when $n \geq 2 d$ it may be required further that $x$ and $y$ are estranged in the sense that they do not share a facet [KW]. A $(d, n)$-polytope $P$ 
is $H$-sharp if and only if $\delta(P) \geq n-d$, and the pair $(d, n)$ is $H$-sharp if and only if $\Delta(d, n) \geq n-d$.

Demonstrating the $H$-sharpness of a pair $(d, n)$ amounts to producing an $H$-sharp $(d, n)$-polytope. Such a polytope with diameter greater than $n-d$ would of course disprove the Hirsch conjecture, but when $n>2 d$ it has been difficult even to produce $(d, n)$-polytopes $P$ for which $\delta(P)$ is equal to $n-d$. For example, of the 1142 combinatorial types of $(4,9)$-polytopes catalogued by Altshuler et al. [ABS], only one (first constructed in [KW]) has diameter 5. Here, as in [HK], that one is denoted by $Q_{4}$.

In the past, polytopes showing the $H$-sharpness of pairs $(d, n)$ with $n>2 d$ have all arisen from $Q_{4}$ by means of elementary wedging and product constructions. The constructions in this paper are also based ultimately on $Q_{4}$, but they use the following additional construction tools:

(i) successive application of truncations of vertices and wedging over facets, extending the methods of $[\mathrm{HK}]$;

(ii) a procedure for blending two $d$-polytopes to form a third one (introduced by Barnette [B] and used by Adler [A] to obtain a general lower bound for $\Delta(d, n))$.

The use of these tools greatly extends the list of pairs $(d, n)$ that are known to be $H$ sharp, and leads also to an improvement of Adler's bound. Figure 1 provides a graphic overview of our results. The most striking new result is the fact that when $d \geq 14$, the pair $(d, n)$ is $H$-sharp for all $n>d$.

Our use of the convenient term $H$-sharp does not imply a belief that the Hirsch conjecture is correct. Indeed, we suspect that $\Delta(d, n)>n-d$ for all sufficiently large $d$ and $n-d$. In view of this belief, it has in the past been irritating that there were so few pairs $(d, n)$ for which $\Delta(d, n)$ was known even to attain the conjectured bound $n-d$. The present note relieves some of the irritation, but it does not add to the list of pairs $(d, n)$ for which the value of $\Delta(d, n)$ is known precisely. Those are still as follows: $\Delta(2, n)=\lfloor n / 2\rfloor, \Delta(3, n)=\lfloor 2 n / 3\rfloor-1[\mathrm{~K}], \Delta(4,9)=5[\mathrm{KW}], \Delta(4,10)=5$ and $\Delta(5,11)=6[\mathrm{G}]$.

In [D1] and [D2], the Hirsch conjecture was stated not only for polytopes but also for convex polyhedra that may be unbounded. However, with $\Delta_{u}(d, n)$ denoting the maximum diameter in the unbounded case, it was shown in [KW] that $\Delta_{u}(4,8)=5$ and consequently

$$
\Delta_{u}(d, n) \geq n-d+\min \left(\left\lfloor\frac{d}{4}\right\rfloor,\left\lfloor\frac{n-d}{4}\right\rfloor\right) .
$$

The methods of the present paper can be used to improve this lower bound on $\Delta_{u}(d, n)$, but the details are omitted because there seems to be no plausible or natural version of the Hirsch conjecture for unbounded polyhedra.

\section{Indication of Results}

Some of our findings are summarized in Fig. 1 and in Corollary 7.4. Each row in Fig. 1 corresponds to the dimension $d$ as labeled, and the column labeled $j$ corresponds to $n=2 d+j$. A box or shading in position $(d, j)$ indicates that $\Delta(d, 2 d+j) \geq d+j$ and 


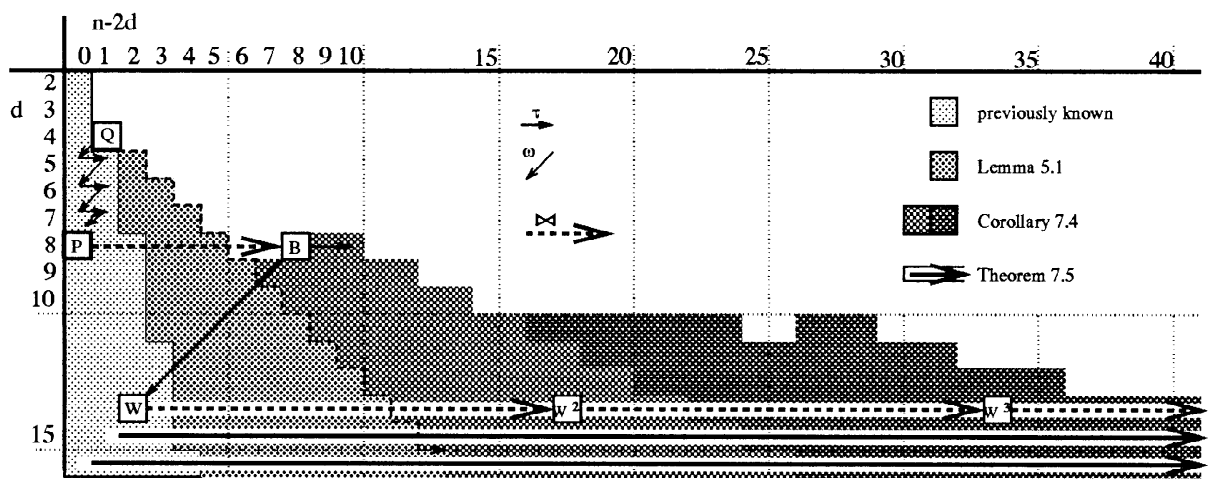

Fig. 1. Some $(d, n)$ for which $\Delta(d, n) \geq n-d$. The previously known examples are indicated by the lighter region on the left. The table indicates a unique $(4,9)$-polytope, which we call $Q_{4}$, of diameter 5 . Using Lemma 5.1 we construct a wedge over $Q_{4}$ and truncate it twice to obtain a $(5,12)$-polytope of diameter 7. (The arrows associated with $\tau, \omega$, and $\bowtie$ indicate the respective operations of truncation, wedging, and blending.) Similarly, once $(d, n)$ is known to be $H$-sharp, products, wedges, and Lemma 5.1 guarantee that $(d+k, n+j+k)$ is $H$-sharp whenever $0 \leq k \leq j \leq 2 k$; that is, once a box is shaded, all the boxes between the lower-left diagonal and lower-right diagonal from this box are shaded. The labeled boxes correspond to the constructions of Theorem 7.3 applied to $Q_{4}$; these constructions are the root of Corollary 7.4, which establishes the $H$-sharpness of the indicated pairs for $d \leq 13$, and of Theorem 7.5, which shows that $(d, n)$ is $H$-sharp for all $n>d \geq 14$.

hence that the Hirsch bound, if correct, is sharp for the pair $(d, 2 d+j)$. The previously known examples are covered by the lighter region on the left. For further details, see the figure's caption and see Corollary 7.4 and Theorem 7.5. The arrows in the 14th, 15th, and 16th rows indicate that the entire rows (and thus all subsequent rows) are $H$-sharp.

\section{Definitions and Notation}

At least two distinct types of "sharpness" for simple polytopes are of interest in connection with the Hirsch conjecture. A $(d, n)$-polytope $P$ is $H$-sharp provided that $\delta(P) \geq n-d$, and $P$ is $\Delta$-sharp provided that $\delta(P)=\Delta(d, n)$. For each pair $(d, n)$ with $n>d$ there are $\Delta$-sharp polytopes; however, there are no $H$-sharp polytopes for $(d, n)=(2, n>4),(3, n>6)$, or $(4,10) . H$-sharpness and $\Delta$-sharpness are equivalent precisely when $\Delta(d, n)=n-d$. The major accomplishment of this paper is the construction of many $H$-sharp polytopes, revealing many pairs $(d, n)$ to be $H$-sharp. Since the constructions involve the interplay of several different methods, some rather technical definitions and notations appear to be required. They are provided by this section.

Let $P$ be a $d$-polytope with diameter $\delta(P)$. For $-1 \leq k \leq d$, let $f^{k}(P)$ denote the set of all $k$-faces of $P$. The members of $f^{0}(P), f^{1}(P), f^{d-2}(P)$, and $f^{d-1}(P)$ are respectively the vertices, edges, ridges, and facets of $P$. For a vertex $x$ of a $(d, n)$-polytope $P$, we define the $H$-set $\mathcal{H}(x)$ for $x$ to be

$$
\mathcal{H}(x)=\mathcal{H}_{P}(x)=\left\{y \in f^{0}(P): \delta_{P}(x, y) \geq n-d\right\}
$$


For a set $X$ of vertices, the $H$-set $\mathcal{H}(X)$ is

$$
\mathcal{H}(X)=\mathcal{H}_{P}(X)=\bigcap_{x \in X} \mathcal{H}(x)
$$

It follows from these definitions that if $\mathcal{H}(x)$ is nonempty, then $x \in \mathcal{H}^{2}(x)$ and $\mathcal{H}^{3}(x)=$ $\mathcal{H}(x)$.

$H$-sets are a special instance of $\delta$-sets. For $x \in f^{0}(P)$ and for any $\delta \geq 0$, the $\delta$-set $D_{P}^{\delta}(x)$ of $x$ in $P$ is defined as follows:

$$
D_{P}^{\delta}(x)=\left\{y \in f^{0}(P): \delta_{P}(x, y) \geq \delta\right\} .
$$

The $H$-set is then $\mathcal{H}_{P}(x)=D_{P}^{n-d}(x)$. We say that two sets $X, Y \subset f^{0}(P)$ form a $\delta$-pair $(X, Y)$ if $\delta_{P}(x, y) \geq \delta$ for each choice of $x \in X$ and $y \in Y$. When $P$ is a $(d, n)$-polytope and $\delta=n-d$, a $\delta$-pair $(X, Y)$ is also called an $H$-pair. When $\delta=\delta(P)$, a $\delta$-pair $(X, Y)$ is called a diametral pair.

(For pairs $(d, n)$ such that $\Delta(d, n)=n-d$, the notion of an $H$-pair can be replaced by the more natural notion of mutually diametral sets. Our lemmas about truncation, wedging, and blending have natural analogues in that context as well.)

For sets $X, Y \subseteq f^{0}(P)$, we define the distance

$$
\delta_{P}(X, Y)=\min _{(x, y) \in X \times Y} \delta_{P}(x, y) .
$$

A short path from $X$ to $Y$ is a path of length $\delta_{P}(X, Y)$ from some $x \in X$ to some $y \in Y$.

We are also concerned with fast edges and slow edges. For a polytope $P$ with $u, v, x \in$ $f^{0}(P)$ and $[u, v] \in f^{1}(P)$, the directed edge $(u, v)$ is fast toward $x$ in $P$ if $\delta(v, x)<$ $\delta(u, x)$. This is equivalent to saying that $(u, v)$ begins a short path from $u$ to $x$, where this means a path of length $\delta_{P}(u, x)$. A directed edge $(u, v)$ that is not fast toward $x$ in $P$ is slow toward $x$ in $P$. If $[u, v] \in f^{1}(P)$ and $\delta_{P}(u, x)=\delta_{P}(v, x)$, then the directed edges $(u, v)$ and $(v, u)$ are both slow toward $x$; in this case, we say that the undirected edge $[u, v]$ is slow toward $x$.

For an $H$-pair $(X, Y)$, an edge $[u, v]$ is fast for $(X, Y)$ if some short path between $X$ and $Y$ contains the edge $[u, v]$; otherwise, the edge $[u, v]$ is slow for $(X, Y)$.

When $P$ is a polytope and $X \subset f^{0}(P)$, we say that $X$ holds a $k$-face of $P$ if there is a $k$-face of $P$ whose vertices all belong to $X$. We denote by $(d, n: h, k)$ the set of all triples $(P, X, Y)$ in which $P$ is an $H$-sharp $(d, n)$-polytope with an $H$-pair $(X, Y)$ such that $X$ holds an $h$-face and $Y$ holds a $k$-face. The collection of all nonempty quadruples $(d, n: h, k)$ is denoted by $\mathcal{T}$.

\section{Wedging}

The wedge $\omega P=\omega_{F} P$ of a $(d, n)$-polytope $P$ over a facet $F \in f^{d-1}(P)$ is a $(d+1, n+$ 1)-polytope, and hence is associated in the figure with the square that is below and to the left of the square associated with $P$. Suppose that $P$ is a $d$-polytope in $\mathbb{R}^{d}$, and $F$ is any face of $P$. In the terminology of [KW], a wedge over $P$ with foot $F$ is a $(d+1)$-polytope $\omega_{F} P$ that is formed by intersecting the "cylinder" $C=P \times[0, \infty[$ with a closed halfspace $J$ in $\mathbb{R}^{d+1}$ such that the intersection $J \cap C$ is bounded and has nonempty interior, 
and the bounding hyperplane $H$ of $J$ is such that $H \cap\left(\mathbb{R}^{d} \times\{0\}\right)=\operatorname{aff}(F) \times\{0\}$. The boundary complex of $\omega_{F} P$ is combinatorially equivalent to the complex formed from the boundary complex of the prism $P \times[0,1]$ by identifying $\{p\} \times[0,1]$ with $(p, 0)$ for each point $p$ of $F$.

In each use of wedging here, the foot of the wedge is a facet of the polytope $P$. In effect, the identification process replaces the facet ( $d$-face) $F \times[0,1]$ of the prism by a ridge $\left((d-1)\right.$-face) $R$ that is a copy of $F$. In the wedge $\omega_{F} P$ there are two facets that contain the ridge $R$, and each of these facets is combinatorially equivalent to $P$. We denote these facets by $B(=P \times\{0\})$ and $T(=P \times\{1\})$ and call them the base and the top of the wedge; thus $R=B \cap T$. Since each vertex of $\omega_{F} P$ is incident to $T$ or $B$, it corresponds naturally to a vertex in $P$. Each vertex $v \in F$ has a unique natural image in the ridge $R$ in $\omega_{F} P$. Each vertex $v \in P \backslash F$ has a natural image in the base $B$ and a second natural image in the top $T$; we denote these images by $v_{b}(=v \times\{0\})$ and $v^{t}$ $(=v \times\{1\})$, respectively. Edges of $\omega P$ of the form $\left[v_{b}, v^{t}\right]$ are called vertical edges.

Each path $\rho$ in $\omega_{F} P$ has a natural image $\pi \rho$ in $P$, obtained by projecting the path onto either the base or top. A path in $P$ has many natural images in $\omega_{F} P$; we can arbitrarily assign each vertex $v$ in the path to one of its images $v_{b}$ or $v^{t}$ and introduce vertical edges as necessary. Given a path from $x$ to $y$ in $P$ and fixed images of the endpoints, this path has a set of tight natural images in $\omega_{F} P$ between these endpoints [HK]. Let $\bar{x} \in\left\{x_{b}, x^{t}\right\}$ and $\bar{y} \in\left\{y_{b}, y^{t}\right\}$, and let $\rho$ be a path from $x$ to $y$ in $P$; then a tight natural image of $\rho$ from $\bar{x}$ to $\bar{y}$ in $\omega_{F} P$ is a path $\bar{\rho}$ from $\bar{x}$ to $\bar{y}$ of minimal length such that $\pi \bar{\rho}=\rho$. In $\omega_{F} P$, if $\bar{x}$ and $\bar{y}$ are coincident either to the base $B$ or to the top $T$, then there is a unique tight natural image $\bar{\rho}$ if and only if the path $\rho$ in $P$ visits the foot $F$ at most once; if one of $\bar{x}$ and $\bar{y}$ is incident to $B$ and the other to $T$, then there is a unique tight natural image in $\omega_{F} P$ if and only if the path in $P$ visits $F$ exactly once. Otherwise, if $\rho$ visits the foot $r \geq 1$ times, then there are $2^{r-1}$ tight natural images.

For any pair of vertices $x$ and $y$, and for any face $F$ of $P$, a path between $x$ and $y$ in $P$ has tight natural images in $\omega_{F} P$, between $x_{b}$ and $y_{b}$, between $x^{t}$ and $y^{t}$, between $x_{b}$ and $y^{t}$, and between $x^{t}$ and $y_{b}$. It is obvious that

$$
\delta_{\omega P}\left(x_{b}, y_{b}\right)=\delta_{\omega P}\left(x^{t}, y^{t}\right)=\delta_{P}(x, y) \quad \text { and that } \quad \delta_{\omega P}\left(x_{b}, y^{t}\right)=\delta_{\omega P}\left(x^{t}, y_{b}\right) .
$$

We make frequent use of the fact that these latter numbers are both equal to $\delta_{P}(x, y)$ if and only if some short path in $P$ from $x$ to $y$ visits $F$, and they are otherwise equal to $\delta_{P}(x, y)+1$. If $P$ is a $(d, n)$-polytope and $F$ is a facet of $P$, then the wedge $\omega_{F} P$ is a $(d+1, n+1)$ polytope, and if $P$ is $H$-sharp, then so is $\omega_{F} P$.

Lemma 3.1. Let $P$ be $a(d, n)$-polytope, let $F$ be a facet of $P$, and let $\omega P=\omega_{F} P$. Then $\delta(\omega P)=\delta(P)$ if and only if, for each diametral pair of vertices $x$ and $y$ of $P$, some short path between $x$ and $y$ visits $F$. In the remaining case, $\delta(\omega P)=\delta(P)+1$.

Proof. The wedging lemma in [HK] tells us that each short path between $x_{b}$ and $y^{t}$ in $\omega P$ is the tight natural image of a short path between $x$ and $y$ in $P$, and consequently $\delta_{\omega P}\left(x_{b}, y^{t}\right)=\delta_{P}(x, y)$ if and only if some short path between $x$ and $y$ visits $F$; otherwise every short path between $x_{b}$ and $y^{t}$ must use a vertical edge. No more than one vertical edge is required. The vertical edge makes each short path in $\omega P$ one longer than its 
natural image in $P$, in which case $\delta_{\omega P}\left(x_{b}, y^{t}\right)=\delta_{P}(x, y)+1$. By considering the natural images in $P$ of short paths between diametral vertices in $\omega P$, we see immediately that $\delta(\omega P) \leq \delta(P)+1$.

If there is a diametral pair of vertices $x$ and $y$ in $P$ such that no short path between them visits $F$, then $\delta(\omega P) \geq \delta(P)+1$; thus in this case $\delta(\omega P)=\delta(P)+1$.

In case there is no such diametral pair, then for each diametral pair $x$ and $y$ some short path $\rho$ between them visits $F$. Then each tight natural image of $\rho$ in $\omega P$ is of length $\delta(P)$ and hence $\delta_{\omega P}\left(x_{b}, y^{t}\right)=\delta(P)$. In this case, $\delta(\omega P)=\delta(P)$.

To track $H$-pairs under wedging, we extend the wedge notation to sets of vertices. For $X \subset f^{0}(P), \omega_{F}(X)$ is defined to be the set of all natural images of elements of $X$ in $\omega_{F} P$; that is, $\omega_{F}(X)=X_{b} \cup X^{t}$. Suppressing the name of the polytope $P$ simplifies the notation, and the identity of $P$ will always be clear from context. Note that the intersection $X_{b} \cap X^{t}$ consists, in effect, of the members of $X$ that are incident to the facet $F$, and if there are no such members, then $\omega_{F}(X)$ consists in effect of two disjoint copies of $X$.

The next lemma and its corollary demonstrate that wedging can be used to increase the dimensions of the faces held by an $H$-pair. The nicer result is that a wedge can increase the dimension of the two faces simultaneously, providing a map from $\left(d, n: h_{1}, h_{2}\right)$ to $\left(d+1, n+1: h_{1}+1, h_{2}+1\right)$; this map requires only that $n>2 d$. To see this, consider any two vertices, one from each of the $h_{i}$-faces held by an $H$-pair. There are at most $2 d$ facets incident to at least one of these two vertices, and wedging over any remaining facet will increase the dimensions of both held faces. By iterating this operation, we obtain polytopes with $H$-pairs holding faces of relatively high dimension, necessary for effective use of truncation and blending, e.g., the Lemma 4.2 and Corollary 6.3 below.

Lemma 3.2. If $(X, Y)$ is an $H$-pair in a $(d, n)$-polytope $P$, and $F$ is a facet of $P$, then $\left(\omega_{F}(X), \omega_{F}(Y)\right)$ is an $H$-pair in the $(d+1, n+1)$-polytope $\omega P=\omega_{F} P$.

Proof. For each pair $(x, y) \in X \times Y$, the tight natural images of short paths between $x$ and $y$ are of length either $\delta_{P}(x, y)$ or $\delta_{P}(x, y)+1$; however, $\omega P$ is a $(d+1, n+1)$ polytope, and $\delta_{P}(x, y) \geq n-d$ by hypothesis.

Many of the polytopes constructed below result from iterated wedging, so we introduce the concise notation $\omega^{k} P$ to denote a $k$-fold wedge over $P$. When $x$ and $y$ are diametral vertices of a $(d, n)$-polytope $P$ with $n>2 d$, at least $n-2 d$ facets of $P$ miss both $x$ and $y$. For each choice $F_{1}, \ldots, F_{k}$ of $k$ such facets, the $k$-fold wedge can be defined inductively by

$$
\omega^{k} P=\omega_{\omega^{k-1} F_{k}} \omega^{k-1} P
$$

Since our main results do not depend on the choice and the order of these $k$ facets incident to neither $x$ nor $y$, we may usually regard $\omega^{k} P$ as denoting any $(d+k, n+k)$-polytope that is formed by successive wedging, in some order, over (the images of) $k$ facets of $P$ that miss both $x$ and $y$.

It follows from Lemma 3.2 that if $P$ is an $H$-sharp $(d, n)$-polytope with $n>2 d$ and 
$H$-pair $(X, Y)$, then, for all $k \leq n-2 d, \omega^{k} P$ is an $H$-sharp $(d+k, n+k)$-polytope with $H$-pair $\left(\omega^{k} X, \omega^{k} Y\right)$.

Corollary 3.3. If $(P, X, Y) \in\left(d, n: h_{1}, h_{2}\right)$ and if there are $k$ facets $F_{1}, \ldots, F_{k}$ such that the sets $X \backslash f^{0}\left(F_{i}\right)$ and $Y \backslash f^{0}\left(F_{i}\right)$ are nonempty for each $F_{i}$, then

$$
\left(\omega^{k} P, \omega^{k} X, \omega^{k} Y\right) \in\left(d+k, n+k: h_{1}+k, h_{2}+k\right) .
$$

\section{In particular,}

(i) if $(d, n) \in \mathcal{S}$, then $(d+k, n+k: k, k) \in \mathcal{T}$ for all $0 \leq k \leq n-2 d$;

(ii) if $\left(d, n: h_{1}, h_{2}\right) \in \mathcal{T}$, then $\left(d+k, n+k: h_{1}+k, h_{2}+k\right) \in \mathcal{T}$ for all $0 \leq k \leq n-2 d$.

Proof. Since the sets $X \backslash f^{0}\left(F_{i}\right)$ and $Y \backslash f^{0}\left(F_{i}\right)$ are nonempty for each $F_{i}$, wedging over the image of $F_{i}$ increases the dimension of the faces held in $X$ and in $Y$. If $(d, n) \in \mathcal{S}$, there exists $(P, x, y) \in(d, n: 0,0)$ such that $x$ and $y$ are estranged; thus there are $n-2 d$ facets incident to neither $x$ nor $y$, and the stated result (i) follows. For (ii), let $x$ be a vertex of the $h_{1}$-face held by $X$ and let $y$ be a vertex of the $h_{2}$-face held by $Y$; there are at least $n-2 d$ facets incident to neither $x$ nor $y$, and the $k$-fold wedge over any $k$ of these establishes the result.

\section{Truncation}

To truncate a $(d, n)$-polytope $P$ at a vertex $v$, we form the intersection $\tau_{v} P$ of $P$ with any closed half-space that misses $v$ and whose bounding hyperplane passes strictly between $v$ and the remaining vertices of $P$. Note that since $P$ is simple, $\tau_{v} P$ is a $(d, n+1)$ polytope with new facet $\tau(v)$ and $d-1$ additional vertices. Combinatorially, the vertex $v$ is replaced by a $(d-1)$-simplex $\Sigma(v)$ with one of its vertices on each edge incident to $v$. For example, if $u$ is a neighbor of $v$ in $P$, then in $\tau_{v} P, \sigma(u)$ is a vertex in $\Sigma(v)$ whose neighbors are the $d-1$ other vertices in $\Sigma(v)$ and $u$.

For a subset $Y \subset f^{0}(P)$, we denote by $\sigma(Y)$ the set

$$
\sigma(Y)=\left\{\sigma(y) \in f^{0}(\tau P): y \in Y\right\} .
$$

Note that $\sigma(Y)$ may be empty, and it is no larger than $Y$; only those $y \in Y$ that are neighbors of $v$ will have corresponding elements in $\sigma(Y)$. Since $\Sigma(v)$ is a $(d-1)$ simplex and $\sigma(Y) \subset f^{0}(\Sigma(v)), \sigma(Y)$ is the set of vertices of some simplex.

Paths in $\tau_{v} P$ have natural images in $P$, obtained by mapping each $\sigma(w)$ to $v$; and each path $\rho$ in $P$ has a unique tight natural image in $\tau_{v} P$, which is the path of minimum length in $\tau_{v} P$ whose natural image in $P$ is $\rho$; if $v$ is an endpoint in $\rho$, then there is a unique tight natural image for each choice of $\sigma(w)$ for the corresponding endpoint.

The next two lemmas make rigorous the observation that not only does truncation at a vertex of a $k$-face $F$ held in an $H$-pair produce another $H$-sharp polytope, but the $(k-1)$-simplex given by the truncation of $F$ at this vertex is held by an $H$-pair in the resulting polytope. Whenever $k-1>0$ we can repeat this process. Thus if a $k$-face 
is held in an $H$-pair, we can truncate any vertex of this $k$-face to obtain an $H$-sharp polytope with a $(k-1)$-face held in an $H$-pair; then we can truncate any vertex of this $(k-1)$-face, then any vertex of the resulting $(k-2)$-face, and so on up to $k$ times.

Lemma 4.1. Let $P$ be an $H$-sharp $(d, n)$-polytope with $H$-pair $(X, Y)$. For $y \in Y$, let $\tau P=\tau_{y}(P)$. If $\sigma(Y)$ is nonempty, then $\delta(\tau P) \geq n-d+1$, and $(X, \sigma(Y))$ is an H-pair in $\tau P$.

Proof. Let $x \in X$ and $\sigma(w) \in \sigma(Y)$, thus $w \in Y$. Any short path from $x$ to $\sigma(w)$ in $\tau P$ must arrive at $\sigma(w)$ either via the edge $[w, \sigma(w)]$ or via the edges $[u, \sigma(u), \sigma(w)]$ for some neighbor $u$ of $y$ in $P$. In either case, the length of the path is increased by one. Since $(X, Y)$ is an $H$-pair in $P$, for each $\sigma(w) \in \sigma(Y)$ and each $x \in X$ we have $\delta_{P}(x, \sigma(w)) \geq n-d+1$ in the $(d, n+1)$-polytope $\tau P$; thus $(X, \sigma(Y))$ is an $H$-pair in $\tau P$.

For $(P, X, Y) \in\left(d, n: h_{1}, h_{2}\right)$, we want to truncate repeatedly in (the images of) the faces held by $X$ and $Y$. We define the $k$-fold truncation in $X, \tau_{X}^{k} P$, inductively as

$$
\tau_{X}^{k} P=\tau_{x \in \sigma^{k-1} X}\left(\tau_{X}^{k-1} P\right),
$$

and

$$
\sigma^{k} X=\sigma\left(\sigma^{k-1} X\right),
$$

in which we first truncate at a vertex of an $h_{1}$-face held by $X$. The polytope $\tau_{Y}^{k} P$ is defined similarly, and we extend this notation by using $\tau_{X}^{k_{1}} \tau_{Y}^{k_{2}} P$ to denote the result of any $k_{1}$-fold truncation in $X$ and $k_{2}$-fold truncation in $Y$, taken in any order. As with $k$-fold wedging, $k$-fold truncation specifies a class of polytopes, depending on the vertices chosen for truncation; since our major results do not depend on this choice, if $(P, X, Y) \in(d, n$ : $\left.h_{1}, h_{2}\right)$, we use $\tau_{X}^{k_{1}} \tau_{Y}^{k_{2}} P$ to denote any $\left(d, n+k_{1}+k_{2}\right)$-polytope obtained by truncating $P k_{1}$ times in the $h_{1}$-face held by $X$ and $k_{2}$ times in the $h_{2}$-face held by $Y$.

Lemma 4.2. Let $(P, X, Y) \in(d, n: h, k)$. Then, for all $0 \leq i \leq h$ and all $0 \leq j \leq k$,

$$
\left(\tau_{X}^{i} \tau_{Y}^{j} P, \sigma^{i} X, \sigma^{j} Y\right) \in(d, n+i+j: h-i, k-j) .
$$

Thus $(d, n+i) \in \mathcal{S}$ for all $0 \leq i \leq h+k$.

Proof. It suffices to show that if $(P, X, Y) \in(d, n: h, k)$ and $v$ is a vertex incident to an $h$-face held by $X$, then $\left(\tau_{v} P, \sigma(X), Y\right) \in(d, n+1: h-1, k)$. Let $F$ be an $h$-face held by $X$ and let $v \in f^{0}(F)$. Truncating $P$ at $v$ introduces a $(d-1)$-simplex $\Sigma(v)$, one of whose facets is the $(h-1)$-simplex $\Sigma_{F}(v)$ introduced by $\tau_{v} F$. Since the argument for the previous lemma applies to each $\sigma_{F}(v), \Sigma_{F}(v)$ is an $(h-1)$-face held by $\sigma(X)$.

That is, if $(P, X, Y) \in(d, n: h, k)$, then we can truncate in $X$ up to $h$ times and in $Y$ up to $k$ times, producing $H$-sharp polytopes from $(d, n)$ to $(d, n+h+k)$.

In this paper we have truncated at vertices for convenience. Similar results hold when the vertex truncations are replaced by truncations at other faces. With $\left(P, X_{1}, X_{2}\right) \in$ 
$\left(d, n: h_{1}, h_{2}\right)$, let $F$ be any $k$-face $(0 \leq k<d-1)$ that contains a $j_{i}$-face of the $h_{i}$-face held by $X_{i}$, where $-1 \leq j_{i}<h_{i}$ but not $j_{1}=j_{2}=-1$. Then

$$
\left(\tau_{F} P, \sigma\left(X_{1}\right), \sigma\left(X_{2}\right)\right) \in\left(d, n+1: h_{1}-j_{1}-1, h_{2}-j_{2}-1\right) .
$$

(When $j_{i}=-1$, we here take $\sigma\left(X_{i}\right)=X_{i}$.) These truncations lead to additional $H$-sharp polytopes, but they do not provide any new $H$-sharp pairs $(d, n)$.

\section{The First Peak}

In Fig. 1 the columns are indexed by $n-2 d$. The prism $P \times I$ is a $(d+1, n+2)$-polytope, which, in the figure, corresponds to the adjacent square below that for $P$. The prism is a special case of a product; in general, the product $P_{1} \times P_{2}$ is a $\left(d_{1}+d_{2}, n_{1}+n_{2}\right)$-polytope with diameter

$$
\delta\left(P_{1} \times P_{2}\right)=\delta\left(P_{1}\right)+\delta\left(P_{2}\right) .
$$

The product $P_{1} \times P_{2}$ is $H$-sharp if and only if both $P_{1}$ and $P_{2}$ are $H$-sharp, and hence the prism $P \times I$ is $H$-sharp if and only if $P$ is.

If any square in the table corresponds to an $H$-sharp pair $(d, n)$, then by prisms and wedges, so do all the squares $(d+k+j, n+2 k+j)$ for $k, j \geq 0$. These lie between a lower-left diagonal from $(d, n)$ and the remainder of the column below the square for $(d, n)$. Starting with the cubes and $Q_{4}$, we obtain a narrow peak of $H$-sharp pairs against the left side of Fig. 1. Additionally, we can take products with $Q_{4}$, which slowly widens the peak as we descend: $Q_{4} \times Q_{4}$ is an $(8,18)$-polytope of diameter 10 , thus all pairs $(d, 2 d+2)$ are $H$-sharp for $d \geq 8$. Generally for each $k \geq 1, H$-sharpness of the pair $(4 k, 9 k)$ follows from consideration of the $k$-fold product of $Q_{4}$ with itself, and consequently all pairs $(d, 2 d+k)$ are $H$-sharp for $d \geq 4 k$.

Our first set of new results broadens this first peak in Fig. 1 by using $Q_{4}$ in the truncation and wedging lemmas given above.

Lemma 5.1. If $(d, n) \in \mathcal{S}$ and $n>2 d$, then $(d+1, n+3) \in \mathcal{S}$.

Proof. Let $P$ be an $H$-sharp $(d, n)$-polytope with $n>2 d$ and estranged diametral vertices $x$ and $y$. Since $n>2 d$, we can take the foot $F$ for $\omega P$ to be incident to neither $x$ nor $y$. By Lemma 3.2, the sets $X=\left\{x_{b}, x^{t}\right\}$ and $Y=\left\{y_{b}, y^{t}\right\}$ form an $H$-pair in $\omega P$. Hence $(\omega P, X, Y) \in(d+1, n+1: 1,1)$, and by Lemma $4.2 \tau_{X} \tau_{Y} \omega P$ is an $H$-sharp $(d+1, n+3)$-polytope.

Starting from $Q_{4}$, we have $(4,9) \in \mathcal{S}$ and thus conclude that $(4+k, 9+j) \in \mathcal{S}$ for all $k \geq 1$ and $k \leq j \leq 3 k$. Equivalently, $(d, 2 d+k) \in \mathcal{S}$ for all $d \geq k+3$. This construction is much more aggressive (i.e., moves to the right in the table more quickly) than does the formation of products. 




Fig. 2. Schematic of the fast-slow blend $\left(P_{1}, x_{1}\right) \bowtie\left(P_{2}, x_{2}\right)$. Illustrated are the edges incident to $x_{1}$ in $P_{1}$ and incident to $x_{2}$ in $P_{2}$, and the neighbors of $x_{1}$ and $x_{2}$ in $P_{1}$ and $P_{2}$, respectively. Of the blended edges, the fast edges from $x_{1}$ toward $Y_{1}$ and from $x_{2}$ toward $Y_{2}$ are indicated by solid line segments, the slow edges by dashed line segments.

\section{Blending}

Our final tool is a refinement of a polytope-blending operation $P_{1} \bowtie P_{2}$ introduced by Barnette [B] and used by Adler [A] to investigate diameters. For $i=1,2$, let $x_{i}$ be a vertex of a $\left(d, n_{i}\right)$-polytope $P_{i}$. The idea behind the blending operation $\bowtie$ is to truncate $P_{i}$ at $x_{i}$ and then to create a new simple $d$-polytope $P_{1} \bowtie P_{2}$ by identifying the facet $\tau\left(x_{1}\right)$ of $P_{1}$ with the facet $\tau\left(x_{2}\right)$ of $P_{2}$ in such a way that each truncated facet of $P_{1}$ blends into a truncated facet of $P_{2}$. The next three paragraphs provide a specific geometric construction of a blend of $P_{1}$ and $P_{2}$ (Fig. 2).

Let $H_{i}$ be a hyperplane in $\mathbb{R}^{d}$ such that $H_{i} \cap P_{i}=\left\{x_{i}\right\}$, and let $U_{i}$ denote the image of $P_{i}$ under a projective transformation that carries $H_{i}$ into the hyperplane at infinity. Then $U_{i}$ is an unbounded simple polyhedron with $n_{i}$ facets, and the $d$ edges of $P_{i}$ incident to $x_{i}$ are carried into $d$ parallel rays. By intersecting $U_{i}$ with a closed half-space whose bounding hyperplane $G_{i}$ is perpendicular to these rays, we obtain a $\left(d, n_{i}+1\right)$-polytope $V_{i}$ in which the new facet $S_{i}$ is a $(d-1)$-simplex that replaces the vertex $x_{i}$ of $P_{i}$.

The next step is to subject $V_{i}$ to an affine transformation which, preserving the perpendicularity to $G_{i}$ of the edges of $V_{i}$ with just one end in $S_{i}$, carries $S_{i}$ onto a regular $(d-1)$-simplex of edge-length 1 . Having done this, we apply rigid motions to bring $S_{1}$ and $S_{2}$ into coincidence on some hyperplane, with $V_{1}$ and $V_{2}$ in opposite half-spaces.

Now, finally, set $P=V_{1} \cup V_{2}=P_{1} \bowtie P_{2}$. Then each of the $d(d-2)$-faces of $S_{i}$ is the intersection of $S_{i}$ with one other facet of $V_{i}$, and these two facets blend together to form a single facet of $P$. Each edge incident to $x_{1}$ in $P_{1}$ is blended together with an edge incident to $x_{2}$ in $P_{2}$ to form a single edge in $P_{1} \bowtie P_{2}$; these edges in $P_{1} \bowtie P_{2}$ together with all faces incident to them form the waist of $P_{1} \bowtie P_{2}$.

In order to fix the combinatorial type of the blended polytope $P_{1} \bowtie P_{2}$, it is not sufficient to specify merely the two "component" polytopes that are to be blended to form $P_{1} \bowtie P_{2}$ and the edges that are involved in the blending operation. We must also specify a permutation $\pi$ that describes the pairing of the $d$ facets $F_{1}, \ldots, F_{d}$ incident to $x_{1}$ with the facets $G_{1}, \ldots, G_{d}$ incident to $x_{2}$; the facet $F_{i}$ is blended with facet $G_{\pi i}$ to form a facet in the waist of $P_{1} \bowtie P_{2}=\left(P_{1}, x_{1}\right) \bowtie_{\pi}\left(P_{2}, x_{2}\right)$. Since the facets $S_{i}$ are regular simplices of the same size, every permutation is permissible, and distinct permutations produce distinct blends, up to any symmetries within or between $P_{1}$ and $P_{2}$. 
We can identify $f^{0}\left(P_{1}\right) \backslash\left\{x_{1}\right\}$ with its image in $P_{1} \bowtie P_{2}$ and likewise $f^{0}\left(P_{2}\right) \backslash\left\{x_{2}\right\}$ with its image. These identifications provide us with a bijection $\pi$ between $f^{0}\left(P_{1} \bowtie P_{2}\right)$ and $f^{0}\left(P_{1}\right) \cup f^{0}\left(P_{2}\right) \backslash\left\{x_{1}, x_{2}\right\}$. Via this identification of vertices, we obtain, for paths in $P_{1} \bowtie P_{2}$, natural images in $P_{1}$ and $P_{2}$.

An edge $\left[u_{1}, u_{2}\right]$ in the waist of $P_{1} \bowtie P_{2}$, with $\pi u_{i} \in f^{0}\left(P_{i}\right)$, has the pair of edges $\left[u_{1}, x_{1}\right]$ and $\left[x_{2}, u_{2}\right]$ for its natural image; any other edge of $P_{1} \bowtie P_{2}$ has a unique edge for its natural image. The natural image of a path $\bar{\rho}$ in $P_{1} \bowtie P_{2}$ is the sequence of edges $\rho$ in $f^{1}\left(P_{1}\right) \cup f^{1}\left(P_{2}\right)$ obtained by taking natural images of the edges in the sequence given in $\bar{\rho}$.

If $\bar{\rho}$ does not contain an edge in the waist of $P_{1} \bowtie P_{2}$, then its natural image $\rho$ is a path in one of $P_{1}$ or $P_{2}$, and $\bar{\rho}$ and $\rho$ will have the same length. If $\bar{\rho}$ uses $k \geq 1$ edges in the waist of $P_{1} \bowtie P_{2}$, then the ordered edges in $\rho$ will form two paths, $\rho_{1}$ in $P_{1}$ and $\rho_{2}$ in $P_{2} ; \rho_{i}$ visits $x_{i}$ precisely $k$ times, and the sum of the lengths of $\rho_{1}$ and $\rho_{2}$ is $k$ more than the length of $\bar{\rho}$.

Let $\rho$ be a path from $v$ to $x_{1}$ in $P_{1}$ which visits $x_{1}$ only once, let $\rho$ have terminal edge $\left[u_{1}, x_{1}\right]$, and let $w \in f^{0}\left(P_{2}\right) \backslash\left\{x_{2}\right\}$. Then an extension of $\rho$ to $w$ is any path $\bar{\rho}$ from $v$ to $w$ in $P_{1} \bowtie P_{2}$ such that the natural image of $\bar{\rho}$ starts with $\rho$ in $P_{1}$. A minimal extension of $\rho$ to $w$ is an extension of $\rho$ to $w$ of minimal length.

Combinatorially, the blending of $P_{1}$ and $P_{2}$ is achieved by a pairwise identification of the facets of $P_{1}$ incident to $x_{1}$ with those of $P_{2}$ incident to $x_{2}$. We describe the combinatorics by giving the incidence matrix $M\left(P_{1} \bowtie P_{2}\right)$ in terms of $M\left(P_{1}\right)$ and $M\left(P_{2}\right)$. Recall that for a polytope $P$ with $n$ facets and $m$ vertices, the incidence matrix $M(P)$ is an $n \times m\{0,1\}$-matrix in which $M_{i j}=1$ if and only if facet $i$ and vertex $j$ are coincident.

First, permute the rows and columns of $M\left(P_{1}\right)$ so that the first column corresponds to the vertex $x_{1}$, and the last $d$ rows correspond to the facets to be blended. Similarly permute the rows and columns of $M\left(P_{2}\right)$ so that the first column corresponds to the vertex $x_{2}$, and the first $d$ rows correspond to the facets to be blended, in the order dictated by $M\left(P_{1}\right)$. That is, the facet corresponding to row $n_{1}-d+1$ of $M\left(P_{1}\right)$ will be blended with the facet corresponding to the first row of $M\left(P_{2}\right)$, and so on:

$$
M\left(P_{1}\right)=\left[\begin{array}{ll}
\langle 0\rangle_{n_{1}-d} & W_{1} \\
\langle 1\rangle_{d} & V_{1}
\end{array}\right]_{n_{1} \times m_{1}} \quad \text { and } \quad M\left(P_{2}\right)=\left[\begin{array}{ll}
\langle 1\rangle_{d} & V_{2} \\
\langle 0\rangle_{n_{2}-d} & W_{2}
\end{array}\right]_{n_{2} \times m_{2}} .
$$

Then the incidence matrix for $\left(P_{1}, x_{1}\right) \bowtie\left(P_{2}, x_{2}\right)$ is given by

$$
M\left(P_{1} \bowtie P_{2}\right)=\left[\begin{array}{cc}
W_{1} & \langle 0\rangle \\
V_{1} & V_{2} \\
\langle 0\rangle & W_{2}
\end{array}\right]_{\left(n_{1}+n_{2}-d\right) \times\left(m_{1}+m_{2}-2\right)} .
$$

The waist of $P_{1} \bowtie P_{2}$ is given by the blocks $\left[V_{1} V_{2}\right]$.

The above descriptions apply to the blending of two simple polytopes at any vertices. However, since we want to construct polytopes of large diameter, we consider only blends that are long with respect to certain pairs $\left(\delta_{1}, \delta_{2}\right)$. This refers to blends of the form $\left(P_{1}, x_{1}\right) \bowtie\left(P_{2}, x_{2}\right)$ where $\delta_{i}$ is a known lower bound on $\delta\left(P_{i}\right)$ and the vertices $x_{1}$ and $x_{2}$ are such that $D_{P_{i}}^{\delta_{i}}\left(x_{i}\right)$ is nonempty. Our primary concern is with the Hirsch bound and hence in the case in which $\delta_{i}=n_{i}-d$. In the remainder of the paper, the notations $\left(P_{1}, x_{1}\right) \bowtie\left(P_{2}, x_{2}\right)$ and $P_{1} \bowtie P_{2}$ indicate long blends. 
By using $Q_{4}$, product formation, and the long blending operation, Adler [A] established the following general lower bound:

$$
\Delta(d, n) \geq n-d-\left\lceil\frac{4(n-d)}{5 d}\right\rceil+1 .
$$

To see this, note (as in [A]) that for a long blend, the polytope $P=P_{1} \bowtie P_{2}$ is a $\left(d, n_{1}+n_{2}-d\right)$-polytope with $\delta(P) \geq \delta_{1}+\delta_{2}-1$. Thus, for fixed $d$, there is an $h_{d}$ such that $\Delta(d, n) \geq n-d$ for all $d<n \leq h_{d}$, and if $n$ is in the range

$$
(k-1)\left(h_{d}-d\right)+h_{d}<n \leq k\left(h_{d}-d\right)+h_{d},
$$

a judicious choice of $P_{1}$ and $P_{2}$ yields $\Delta(d, n) \geq n-d-k$ with

$$
k=\left\lceil\frac{n-h_{d}}{h_{d}-d}\right\rceil=\left\lceil\frac{n-d}{h_{d}-d}\right\rceil-1 .
$$

Given the previously known $H$-sharp pairs, as indicated in Fig. 1, Adler could use only $h_{d}=\lfloor 9 d / 4\rfloor$.

In the blend $\left(P_{1}, x_{1}\right) \bowtie\left(P_{2}, x_{2}\right)$, each edge incident to $x_{1}$ in $P_{1}$ is blended with an edge incident to $x_{2}$ in $P_{2}$ to form a single edge in the waist of $P_{1} \bowtie P_{2}$. An edge in the waist of a long blend $P_{1} \bowtie P_{2}$ is either a fast-slow edge, a fast-fast edge, or a slow-slow edge, depending on whether the two edges blended to form it were fast or slow toward $D_{P_{i}}^{\delta_{i}}\left(x_{i}\right)$.

A fast-slow blend is a long blend in which there are no fast-fast edges in the waist. Each fast edge from $x_{1}$ toward $D_{P_{1}}^{\delta_{1}}\left(x_{1}\right)$ is blended with a slow edge from $x_{2}$ toward $D_{P_{2}}^{\delta_{2}}\left(x_{2}\right)$, and each fast edge from $x_{2}$ toward $D_{P_{2}}^{\delta_{2}}\left(x_{2}\right)$ is blended with a slow edge from $x_{1}$ toward $D_{P_{1}}^{\delta_{1}}\left(x_{1}\right)$. There may also be slow edges blended with slow edges, but there are no fast-fast edges. Look again at Fig. 2.

Lemma 6.1. If $P_{1} \bowtie P_{2}=\left(P_{1}, x_{1}\right) \bowtie\left(P_{2}, x_{2}\right)$ is a fast-slow blend, then

$$
\delta\left(P_{1} \bowtie P_{2}\right) \geq \delta_{1}+\delta_{2} .
$$

Proof. Since a fast-slow blend is a long blend, the sets $Y_{1}=D_{P_{1}}^{\delta_{1}}\left(x_{1}\right)$ and $Y_{2}=D_{P_{2}}^{\delta_{2}}\left(x_{2}\right)$ are both nonempty. Let $y_{1} \in Y_{1}$ and $y_{2} \in Y_{2}$, and let $U_{1}$ be the neighbors of $x_{1}$ in $P_{1}$ and let $U_{2}$ be the neighbors of $x_{2}$ in $P_{2}$.

Any short path in $P_{1} \bowtie P_{2}$ between $y_{1}$ and $y_{2}$ has a natural image in $P_{1}$ from $y_{1}$ to $x_{1}$ and another in $P_{2}$ from $x_{2}$ to $y_{2}$. For $\delta_{P_{1} \bowtie P_{2}}\left(y_{1}, y_{2}\right)<\delta_{1}+\delta_{2}$, both of these natural images must be short paths.

Any short path in $P_{1}$ from $y_{1}$ to $x_{1}$ terminates with a fast edge $\left[u_{1}, x_{1}\right]$ for some $u_{1} \in U_{1}$, and $\delta_{P_{1}}\left(y_{1}, u_{1}\right)=\delta_{P_{1} \bowtie P_{2}}\left(y_{1}, u_{1}\right) \geq \delta_{1}-1$. In the waist, there is a unique edge [ $\left.u_{1}, u_{2}\right]$ incident to $u_{1}$. By assumption, $P_{1} \bowtie P_{2}$ is a fast-slow blend, so $\left[x_{2}, u_{2}\right]$ is a slow edge to $y_{2}$ in $P_{2}$, and $\delta_{P_{2}}\left(y_{2}, u_{2}\right)=\delta_{P_{1} \bowtie P_{2}}\left(y_{2}, u_{2}\right) \geq \delta_{2}$. That is, each short path from $y_{1}$ to $x_{1}$ in $P_{1}$ can be minimally extended to a path in $P_{1} \bowtie P_{2}$ from $y_{1}$ to $y_{2}$ of length $\left(\delta_{1}-1\right)+1+\delta_{2}=\delta_{1}+\delta_{2}$. Thus $\delta_{P_{1} \bowtie P_{2}}\left(y_{1}, y_{2}\right) \geq \delta_{1}+\delta_{2}$, and so $\delta\left(P_{1} \bowtie P_{2}\right) \geq$ $\delta_{1}+\delta_{2}$. 
Lemma 6.2. Let $\left(P_{1}, x_{1}\right) \bowtie\left(P_{2}, x_{2}\right)$ be a fast-slow blend of $H$-sharp polytopes $P_{1}$ and $P_{2}$, with respective $H$-pairs $\left(Y_{1},\left\{x_{1}\right\}\right)$ and $\left(Y_{2},\left\{x_{2}\right\}\right)$. Then $P_{1} \bowtie P_{2}$ is $H$-sharp, with $H$-pair $\left(Y_{1}, Y_{2}\right)$.

Proof. Since $P_{1}$ is an $H$-sharp $\left(d, n_{1}\right)$-polytope and $P_{2}$ is an $H$-sharp $\left(d, n_{2}\right)$-polytope, we may take $\delta_{1}=n_{1}-d$ and $\delta_{2}=n_{2}-d$. Then $P_{1} \bowtie P_{2}$ is a $\left(d, n_{1}+n_{2}-d\right)$-polytope, which by the previous lemma has diameter

$$
\delta\left(P_{1} \bowtie P_{2}\right) \geq \delta_{1}+\delta_{2}=n_{1}+n_{2}-2 d .
$$

Thus the fast-slow blend $P_{1} \bowtie P_{2}$ is $H$-sharp if both $P_{1}$ and $P_{2}$ are. Moreover, the proof in the previous lemma shows that $\delta_{P_{1} \bowtie P_{2}}\left(y_{1}, y_{2}\right) \geq n_{1}-d+n_{2}-d$ for each such $\left(y_{1}, y_{2}\right) \in Y_{1} \times Y_{2}$.

Corollary 6.3. If $\left(d, n_{1}: h_{1}, k_{1}\right),\left(d, n_{2}: h_{2}, k_{2}\right) \in \mathcal{T}$ and $h_{1}+h_{2} \geq d$, then $\left(d, n_{1}+\right.$ $\left.n_{2}-d: k_{1}, k_{2}\right) \in \mathcal{T}$.

Proof. For $i=1,2$, let $\left(P_{i}, X_{i}, Y_{i}\right) \in\left(d, n_{i}: h_{i}, k_{i}\right)$, and let $x_{i}$ be a vertex of the $h_{i}$-face held by $X_{i}$. Since $h_{1}+h_{2} \geq d$, we can take $\left(P_{1}, x_{1}\right) \bowtie\left(P_{2}, x_{2}\right)$ to be a fast-slow blend, and the previous lemma yields

$$
\left(P_{1} \bowtie P_{2}, Y_{1}, Y_{2}\right) \in\left(d, n_{1}+n_{2}-d: k_{1}, k_{2}\right) .
$$

If the fast-slow blend of $H$-sharp polytopes $P_{1} \bowtie P_{2}$ is not a counterexample to the Hirsch conjecture, then $\delta\left(P_{1} \bowtie P_{2}\right)=\delta\left(P_{1}\right)+\delta\left(P_{2}\right)$, and $\left(Y_{1}, Y_{2}\right)$ is a diametral pair.

\section{Additional Peaks}

This section uses blending, truncation, and wedging to create a second peak from the first one in the table, a third from the second, and so on until the peaks blend together into broad plateaus.

Because of their frequent occurrence in the blendings below, we introduce a special notation to designate $H$-sharp $(d, 2 d)$-polytopes that have $H$-pairs holding faces of fairly high dimension. The symbol $P_{d: h, k}$ denotes an arbitrary $(d, 2 d)$-polytope with an $H$-pair $(X, Y)$ such that $\left(P_{d: h, k}, X, Y\right) \in(d, 2 d: h, k)$.

Lemma 7.1. For each $d \geq 5$ and each $k$ with $1 \leq k \leq d-4$, there is a triple $\left(P_{d: k, d-3-k}, X, Y\right) \in(d, 2 d: k, d-3-k)$.

Proof. For $d=5$, we have

$$
\left(P_{5: 1,1}, X, Y\right)=\left(\omega Q_{4}, \omega\{x\}, \omega\{y\}\right) \in(5,10: 1,1) .
$$

Now it suffices to note the inductive step that if $(P, X, Y) \in\left(d, 2 d: h_{1}, h_{2}\right)$ with $h_{1}>0$, then

$$
\left(\tau_{X} P, \sigma(X), Y\right) \in\left(d, 2 d+1: h_{1}-1, h_{2}\right)
$$


and

$$
\left(\omega \tau_{X} P, \omega \sigma(X), \omega Y\right) \in\left(d+1,2 d+2: h_{1}, h_{2}+1\right) .
$$

The polytopes $P_{d}$ of [HK] provide the extreme example $P_{d: d-4,1}$; and having a $(d-4)$ face held by one member of an $H$-pair enables us to perform blends on any polytope in which one member of an $H$-pair holds a 4 -face. We can produce polytopes with these 4-faces either by alternately wedging and truncating as above, or by simply wedging when $n-2 d$ is large enough.

Lemma 7.2. Let $(Q, X, Y) \in\left(d, n: h_{1}, h_{2}\right)$, and let $k=\max \left\{4-h_{1}, 4-h_{2}, 0\right\}$. If $n-2 d \geq k$, then

(i) $(d+k, n+k+j) \in \mathcal{S}$ for $0 \leq j \leq 8$;

and if $d+k \geq 5$, then

(ii) $(d+k, n+d+2 k+j) \in \mathcal{S}$ for $0 \leq j \leq 5$;

(iii) $(d+k, n+2 d+3 k+j) \in \mathcal{S}$ for $0 \leq j \leq 2$.

Proof. If $n-2 d \geq k$, we can apply Corollary 3.3 to the $k$-fold wedge $\omega^{k} Q$. Since each of $X$ and $Y$ holds a (4-k)-face, in $\omega^{k} Q$ the sets $\omega^{k}(X)$ and $\omega^{k}(Y)$ form an $H$-pair and each of these sets holds a 4-face. Truncating the vertices of both of these 4-faces, we obtain (i).

Now make a fast-slow blend $\omega^{k} Q \bowtie P_{d+k: d+k-4,1}$, thus forming an $H$-sharp $(d+$ $k, n+d+2 k)$-polytope with an $H$-pair $(X, Y)$ in which $X$ holds a 4 -face and $Y$ holds a 1-face. We can truncate this polytope in $X$ and $Y$ up to five times, establishing (ii).

Finally for (iii), make a fast-slow blend

$$
P_{d+k: 1, d+k-4} \bowtie \omega^{k} Q \bowtie P_{d+k: d+k-4,1} ;
$$

this is an $H$-sharp $(d+k, n+2 d+3 k)$-polytope with an $H$-pair $(X, Y)$ in which each set holds a 1-face. We can truncate this polytope once in $X$ and once in $Y$ to produce $H$-sharp polytopes.

Theorem 7.3. If $(d, n) \in \mathcal{S}$ with $n>2 d$, then

(i) $(d+1, n+1),(d+1, n+2),(d+1, n+3) \in \mathcal{S}$;

(ii) for $0 \leq k \leq d+1,(2 d, n+3 d-k) \in \mathcal{S}$;

(iii) $(2 d, 2 n+2 d-2),(2 d, 2 n+2 d-1),(2 d, 2 n+2 d) \in \mathcal{S}$;

(iv) for $k \geq 1$ and $0 \leq j \leq 4 d-2,(4 d-2,4 d-2+(2 n-2) k) \in \mathcal{S}$;

(v) for $k \geq 1$ and $0 \leq j \leq 4 d-5$, $(4 d-2,8 d-4+(2 n-2) k+j) \in \mathcal{S}$;

(vi) for $k \geq 1$ and $0 \leq j \leq 4 d-8$, $(4 d-2,12 d-6+(2 n-2) k+j) \in \mathcal{S}$.

Proof. For each pair indicated, we produce an $H$-sharp polytope. Start with an $H$-sharp $(d, n)$-polytope $Q$, and let $X$ and $Y$ be an $H$-pair of $Q$. Then apply wedging, truncation, and blending in the ways described below. (Refer to Lemma 3.2 for wedges, Lemma 4.2 for truncations, and Lemma 6.2 for blends.) For the polytopes $P_{d: h, k}$ that are involved 
in this proof and the next one, we always assume that $h \leq k$ and we use $X_{P}$ and $Y_{P}$ to denote the indicated $H$-pair; thus $X_{P}$ holds the face of lower dimension.

(i) $(Q, X, Y) \in(d, n: 0,0)$, so $(\omega Q, \omega X, \omega Y) \in(d+1, n+1: 1,1)$, and by Lemma 4.2, $(d+1, n+1),(d+1, n+2),(d+1, n+3) \in \mathcal{S}$.

(ii) As in the extreme example of $P_{d: d-4,1}$, we form $P=\left(\omega \tau_{Y}\right)^{d-1} \omega Q$, with all wedges over the truncated top of the previous wedge. Let $\bar{X}$ and $\bar{Y}$ be the images in $P$ of $X$ and $Y$. Now $(P, \bar{X}, \bar{Y}) \in(2 d, n+2 d-1: d, 1)$. For $0 \leq k \leq d$,

$$
\left(\tau_{X}^{k} \tau_{Y} P, \sigma^{k} X, \sigma Y\right) \in(2 d, n+2 d-1+k: d-k, 0) .
$$

(iii) Continuing with the $P$ of part (ii), $(P, \bar{X}, \bar{Y}) \in(2 d, n+2 d-1: d, 1)$, we take a vertex $x \in \bar{X}$ incident to this $d$-face and form the fast-slow blend $B=(P, x) \bowtie(P, x)$. This blend $B$ is an $H$-sharp $(2 d, 2 n+2 d-2)$-polytope with an $H$-pair $\bar{Y}_{1}$ and $\bar{Y}_{2}$, each of which holds an edge. Thus $\left(B, \bar{Y}_{1}, \bar{Y}_{2}\right) \in(2 d, 2 n+2 d-2: 1,1)$, and, by Lemma 4.2, $(2 d, 2 n+2 d-2),(2 d, 2 n+2 d-1),(2 d, 2 n+2 d) \in \mathcal{S}$.

(iv) The wedge $W=W^{1}=\omega^{2 d-2} B$ is an $H$-sharp $(4 d-2,2 n+4 d-4)$-polytope with $H$-pair $\omega^{2 d-2} \bar{Y}_{1}$ and $\omega^{2 d-2} \bar{Y}_{2}$, each of which holds a $(2 d-1)$-face. Thus

$$
\left(W, \omega^{2 d-2} \bar{Y}_{1}, \omega^{2 d-2} \bar{Y}_{2}\right) \in(4 d-2,2 n+4 d-4: 2 d-1,2 d-1) .
$$

Now let $W^{2}=W \bowtie W$, and inductively $W^{k}=W^{k-1} \bowtie W=(W \bowtie)^{k-1} W$. Then

$$
\left(W^{k}, \omega^{2 d-2} \bar{Y}_{1}, \omega^{2 d-2} \bar{Y}_{2}\right) \in(4 d-2,4 d-2+(2 n-2) k: 2 d-1,2 d-1) .
$$

Lemma 4.2 allows up to $4 d-2$ truncations in $W^{k}$ that produce $H$-sharp polytopes.

(v) For $k \geq 1$, we can also form the fast-slow blend $W^{k} \bowtie P_{4 d-2: 2 d-1,2 d-4}$. This $H$-sharp polytope provides the triple

$$
\begin{aligned}
\left(W^{k} \bowtie P_{4 d-2: 2 d-1,2 d-4},\right. & \left.\omega^{2 d-2} \bar{Y}_{1}, X_{P}\right) \\
\in(4 d-2, & 8 d-4+(2 n-2) k: 2 d-1,2 d-4) .
\end{aligned}
$$

We again appeal to Lemma 4.2 to obtain the full result.

(vi) For $k \geq 1$, a fast-slow blend on $W^{k}$ produces the triple

$$
\begin{aligned}
&\left(P_{4 d-2: 2 d-4,2 d-1} \bowtie W^{k} \bowtie P_{4 d-2: 2 d-1,2 d-4},\right.\left.X_{P}, X_{P}\right) \\
& \in(4 d-2,12 d-6+(2 n-2) k: 2 d-4,2 d-4) .
\end{aligned}
$$

Lemma 4.2 establishes the result.

Applying this theorem to $Q_{4}$ justifies the entries in Fig. 1. Corollary 7.4 identifies the relevant polytopes for $d<14$, and Theorem 7.5 establishes that $\Delta(d, n) \geq n-d$ for all $n>d \geq 14$.

Corollary 7.4. Since the $(4,9)$-polytope $Q_{4}$ is $H$-sharp, the following pairs are $H$ sharp:

$$
\begin{array}{llll}
(5, n \leq 12), & (6, n \leq 15), & (7, n \leq 18) \\
(8, n \leq 21), & (8,24 \leq n \leq 26), & (9, n \leq 30), & (10, n \leq 34) \\
(11, n \leq 46), & (11,49 \leq n \leq 51), & (12, n \leq 56), & (13, n \leq 62)
\end{array}
$$


Proof. Apply Theorem 7.3 to the triple $\left(Q_{4},\{x\},\{y\}\right) \in(4,9: 0,0)$. By (i), the set $\mathcal{S}$ includes $(5,10),(5,11)$, and $(5,12)$, by (ii) it includes $(8,16), \ldots,(8,21)$, and by (iii) it includes $(8,24),(8,25)$, and $(8,26)$.

For $d=9,10$, we take the two blends

$$
\left(P_{9: 1,5} \bowtie P_{9: 4,2}, X, Y\right) \in(9,27: 1,2),
$$

and

$$
\left(P_{10: 2,5} \bowtie P_{10: 5,2}, X, Y\right) \in(10,30: 2,2) .
$$

Now apply Theorem 7.3 to $(Q, X, Y) \in(8,24: 1,1)$. Then

$$
\begin{aligned}
\left(\omega^{3} Q, \omega^{3} X, \omega^{3} Y\right) & \in(11,27: 4,4), \\
\left(P_{11: 1,7} \bowtie \omega^{3} Q, X_{P}, Y\right) & \in(11,38: 1,4), \\
\text { and } \quad\left(P_{11: 1,7} \bowtie \omega^{3} Q \bowtie P_{11: 7,1}, X_{P}, X_{P}\right) & \in(11,49: 1,1) ;
\end{aligned}
$$$$
\left(\omega^{4} Q, \omega^{4} X, \omega^{4} Y\right) \in(12,28: 5,5),
$$$$
\left(P_{12: 2,7} \bowtie \omega^{4} Q, X_{P}, \omega^{4} Y\right) \in(12,40: 2,5),
$$

and $\left(P_{12: 2,7} \bowtie \omega^{4} Q \bowtie P_{12: 7,2}, X_{P}, X_{P}\right) \in(12,52: 2,2)$;

$$
\left(\omega^{5} Q, \omega^{5} X, \omega^{5} Y\right) \in(13,29: 6,6),
$$

$$
\left(P_{13: 3,7} \bowtie \omega^{5} Q, X_{P}, \omega^{5} Y\right) \in(13,42: 3,6),
$$

and $\left(P_{13: 3,7} \bowtie \omega^{5} Q \bowtie P_{13: 7,3}, X_{P}, X_{P}\right) \in(13,55: 3,3)$.

Now let $(Q, X, Y) \in(7,18: 0,0)$. Then

$$
\left(\omega^{4} Q, \omega^{4} X, \omega^{4} Y\right) \in(11,22: 4,4),
$$

and

$$
\left(P_{11: 1,7} \bowtie \omega^{4} Q \bowtie P_{11: 7,1}, X_{P}, X_{P}\right) \in(11,44: 1,1) .
$$

Take $(Q, X, Y) \in(9,30: 0,0)$. Then

$$
\left(\omega^{4} Q, \omega^{4} X, \omega^{4} Y\right) \in(13,34: 4,4),
$$

and

$$
\left(P_{13: 1,9} \bowtie \omega^{4} Q \bowtie P_{13: 9,1}, X_{P}, X_{P}\right) \in(13,60: 1,1) .
$$

Lemmas 5.1 and 4.2 demonstrate the $H$-sharpness of the remaining pairs in Fig. 1 for $d<13$.

Theorem 7.5. For all $d \geq 14$ and all $n>d,(d, n) \in \mathcal{S}$.

Proof. It suffices to show that, for all $n \geq 15,(14, n)$ is $H$-sharp. For this, we apply Theorem 7.3 to $Q_{4} \in(4,9)$. Taking $Q=Q_{4}$, the $P$ in (ii) is an $(8,16)$-polytope; the $B$ in (iii) is an $H$-sharp $(8,24)$-polytope with diametral sets $X$ and $Y$, each of which contains the vertices of an edge. The wedge $W$ of (iv) is an $H$-sharp $(14,30)$-polytope, and $W^{k}$ is an $H$-sharp $(14,14+16 k)$-polytope. Truncations of $W^{k}$ yield $H$-sharp $(14,14+16 k+j)$ polytopes for all $k \geq 1$ and all $0 \leq j \leq 14$. The polytopes of part (v) fill the remaining gaps: $W^{k} \bowtie P_{14: 7,4}$ is an $H$-sharp $(14,28+16 k)$-polytope whose truncations yield $H$-sharp $(14,28+16 k+j)$-polytopes for all $k \geq 1$ and all $0 \leq j \leq 11$. 
All the polytopes constructed in this paper were derived from the unique $H$-sharp $(4,9)$-polytope $Q_{4}$. The pair $(4,10)$ is known not to be $H$-sharp, but the $H$-sharpness of the pairs $(4, n \geq 11)$ is unknown. If there is an $H$-sharp $(4,11)$-polytope, then it follows from Corollary 3.3, Lemma 4.2, and Corollary 6.3 that the entire row $d=9$ is $H$-sharp; and if there is an $H$-sharp $(4, n \geq 12)$-polytope, these would demonstrate that the entire row $d=8$ is $H$-sharp, with the possible exceptions of $(8, m)$ for $m=22,23$ and $27 \leq m<n+4$. If there is an $H$-sharp (4, $12 \leq n \leq 18)$-polytope, then the entire row $d=8$ is $H$-sharp.

\section{References}

[A] I. Adler, Lower bounds for maximum diameters of polytopes, Math. Programming Stud., 1:11-19, 1974.

[ABS] A. Altshuler, J. Bokowski, and L. Steinberg, The classification of simplicial 3-spheres with nine vertices into polytopes and nonpolytopes, Discrete Math., 31:115-124, 1980.

[B] D. Barnette, A simple 4-dimensional nonfacet, Israel J. Math., 7:16-20, 1969.

[D1] G. B. Dantzig, Linear Programming and Extensions, Princeton University Press, Princeton, NJ, 1963.

[D2] G. B. Dantzig, Eight unsolved problems from mathematical programming, Bull. Amer. Math. Soc., 70:499-500, 1964.

[G] P. Goodey, Some upper bounds for the diameter of convex polytopes, Israel J. Math., 11:380-385, 1972.

[HK] F. Holt and V. Klee, Counterexamples to the strong $d$-step conjecture for $d \geq 5$, Discrete Comput. Geom., 19:33-46, 1998.

[K] V. Klee, Diameters of polyhedral graphs, Canad. J. Math., 16:602-614, 1964.

[KW] V. Klee and D. W. Walkup, The $d$-step conjecture for polyhedra of dimension $d<6$, Acta Math., 133:53-78, 1967.

Received November 20, 1996, and in revised form March 21, 1997.

Added in proof. Corollary 7.4 has been extended to include eight additional $H$-sharp pairs: $(8,22),(8,23),(11,47),(11,48),(12,57),(13,63),(13,64),(13,65)$. The last four of these were discovered by Kerstin Fritzsche. 